\title{
A RADIAL-VELOCITY CURVE FOR WR 46 (WN3P)
}

\author{
P.M. VEEN, A.M. VAN GENDEREN and M.A.W. VERHEIJEN \\ Sterrewacht Leiden, The Netherlands \\ and \\ K.A. VAN DER HUCHT \\ SRON-Utrecht, The Netherlands
}

\begin{abstract}
As the first result of spectroscopic monitoring of WR46 (HD 104994, WN3p) in 1991, a preliminary radial-velocity curve is presented with $P=6.8 \mathrm{hr}$, confirming the binary nature suggested from photometry.
\end{abstract}

\section{Introduction}

We investigate the spectral and photometric variability of WR46 (WN3p). As shown by van Genderen et al. (1991), the Walraven-photometric lightcurve shows a double-wave with a period of $6.8 \mathrm{hr}$. This fact and the colour changes are indicative of a binary motion. In support of this hypothesis, Verheijen (1991) found indication of a low amplitude radial velocity curve $(K \approx 40 \mathrm{~km} / \mathrm{s})$ in spectra from 1989 . This period was also present in variations of the equivalent widths of the emission lines. It appeared that the flux contents of the various lines varied by more than $10 \%$ and that the variations were more or less in phase with the continuum variations. This variability could be due to binary motion. However, the available spectra do not show spectral features of a companion.

Here, we present a preliminary radial velocity curve by means of crosscorrelation of spectra taken in 1991. This radial-velocity curve has an amplitude of about $100 \mathrm{~km} / \mathrm{s}$.

\section{Data, method and results}

The spectra were taken in three consecutive nights at the ESO $1.5 \mathrm{~m}$ telescope with a Boller \& Chivens spectrograph (3700-7300 $\AA, 3.7 \AA /$ pixel). Cross-correlation has been performed as described by van Kerkwijk (1993a). For each spectrum all the major emission lines have been cross-correlated with the first spectrum of the second night, which is arbitrarily put at phase zero. Any linear shift between the spectra is corrected for by cross-correlation of the interstellar and telluric lines.

Figure 1 shows an overplot of spectral lines of the ions $\mathrm{N} \mathrm{V}$ and $\mathrm{He}$ II from spectra of the second and third night. An arbitrary line is drawn to show the radial velocity variations more clearly and the phase (see above) is indicated. 
Figure 2 shows the radial velocity measurements using all the major emission lines simultaneously. Systematic effects, like profile-changes within a cycle and from cycle to cycle are not yet taken into account. No conclusions can yet be drawn about any phase-shift between lines or differences in amplitude for different lines. However, the general course of a radial-velocity curve with a period equal to the double-wave period in photometry can be clearly identified.

If WR46 is indeed a binary, then the companion is probably of low mass, since it does not show spectral features and the radial velocity curve has a small amplitude. The companion might be a compact star, although the system lacks the copious X-ray luminosity of Cygnus X-3 (Pollock 1987; van Kerkwijk 1993a,b). We will continue with the reduction and analysis of spectra and simultaneous photometry taken by us in 1989 and 1990 .

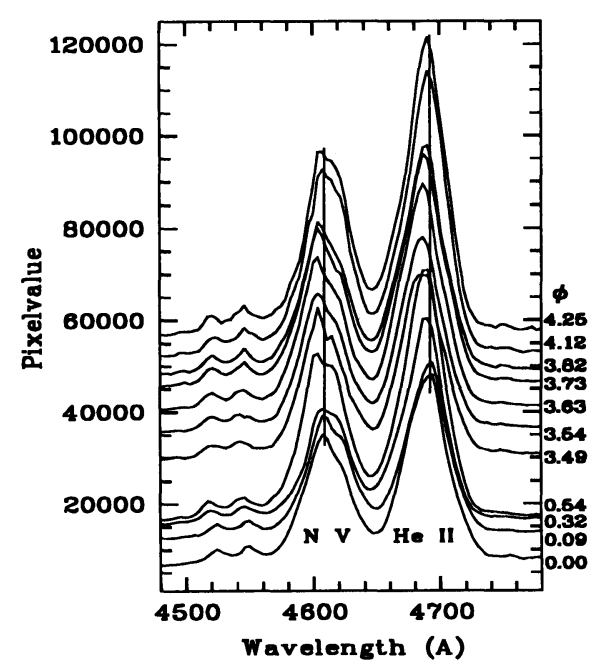

Fig. 1. Spectral variations of WR46.

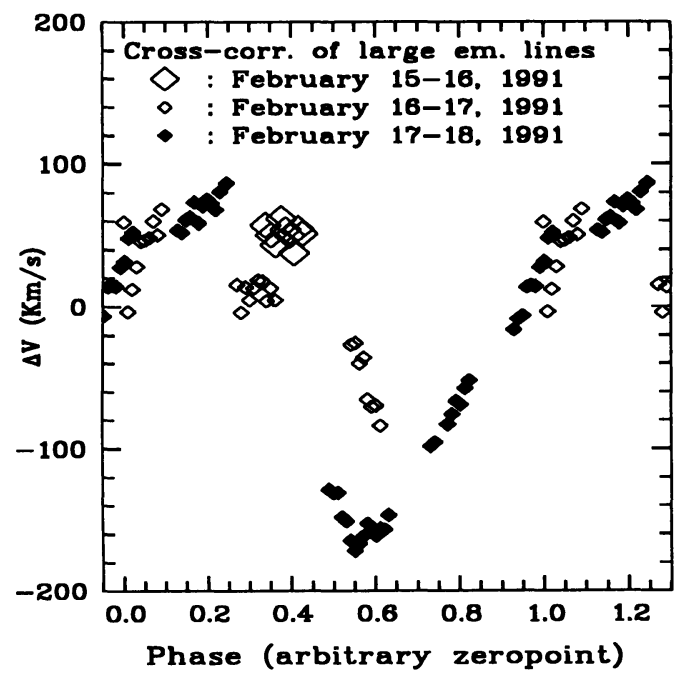

Fig. 2. Radial velocity curve of WR46.

\section{References}

van Genderen, A.M. 1991, in: K.A. van der Hucht \& B. Hidayat (eds.) Wolf-Rayet Stars and Interrelations with Other Massive Stars in Galaxies, Proc. IAU Symp. No. 143 (Dordrecht: Kluwer), p. 129

van Kerkwijk, M.H. 1993a, PhD thesis, University of Amsterdam

van Kerkwijk, M.H. 1993b, A\&A (Letters) 276, L9

Pollock, A.M.T. 1987, $A p J$ 320, 283

Verheijen, M.A.W. 1991, Internal Report, Observatory Leiden 